\title{
Plasma Current Sustainment by RF Power in ECH Start-up Plasma in the TST-2 Spherical Tokamak
}

\author{
Osamu WATANABE, Akira EJIRI, Takashi YAMAGUCHI, Junichi SUGIYAMA, \\ Takuya OOSAKO, Yuuki ADACHI, Kotaro YAMADA, Hiroshi TOJO, Soichiro KAINAGA ${ }^{1)}$, \\ Yuichi TAKASE, Yoshihiko NAGASHIMA, Makoto SASAKI ${ }^{1)}$ and Tetsuya MASUDA \\ Graduate School of Frontier Sciences, The University of Tokyo, Kashiwa 277-8561, Japan \\ ${ }^{1)}$ Graduate School of Sciences, The University of Tokyo, Bunkyo 113-0033, Japan
}

(Received 21 May 2008 / Accepted 19 July 2008)

\begin{abstract}
In the TST-2 spherical tokamak, a non-inductive start-up plasma produced by electron cyclotron heating $(\mathrm{ECH})$ at $2.45 \mathrm{GHz}(3-5 \mathrm{~kW})$ is sustained by RF wave heating at $21 \mathrm{MHz}(10-24 \mathrm{~kW})$. A current jump, which occurs at a certain time when only ECH is used, can be induced earlier by the RF heating, and a constant plasma current is sustained even after the ECH power is turned off. The plasma current is sustained in both hydrogen and deuterium plasmas, as long as the RF power exceeds a threshold level, which is lower for deuterium plasma. The maximum sustainment time by the the RF power alone was $40 \mathrm{~ms}$, limited by the RF pulse length. According to CCD camera images of the total plasma light, the major radii are almost the same, but the RF-sustained plasma has a more peaked profile, compared with the distribution of an ECH-sustained plasma.
\end{abstract}

(c) 2008 The Japan Society of Plasma Science and Nuclear Fusion Research

Keywords: non-inductive current drive, RF heating, spherical tokamak

DOI: $10.1585 /$ pfr.3.049

Non-inductive plasma start-up and sustainment methods are required for spherical tokamak reactors, in which central ohmic solenoid coils should be eliminated [1,2]. In TST-2, electron cyclotron heating (ECH) start-up experiments were performed with a magnetron (EC: $2.45 \mathrm{GHz} /$ $5 \mathrm{~kW}$ ) [3]. EC power is expected to heat electrons at both fundamental and second harmonic electron cyclotron resonance layers. The toroidal magnetic field generated by the capacitor bank discharge decays temporally, so the resonance layers move toward the smaller major radius side. The plasma can be sustained until the fundamental resonance layer reaches the inboard wall. The maximum ECH start-up, plasma current is about $2 \mathrm{kA}$ with the help of the vertical field ramp-up operation. A typical sustained plasma has a major radius of $0.4 \mathrm{~m}$ and a minor radius of $0.18 \mathrm{~m}$.

The RF wave has a frequency of $21 \mathrm{MHz}$ and it is more than ten times higher than hydrogen ion cyclotron frequency. The wave excited by single or double straps can propagate through standard high density TST- 2 plasmas. In the low density start-up plasmas, however, the dominant component becomes evanescent. In the experiments reported in this paper, the RF wave was excited by a single strap, and a minor component with a toroidal mode number of about one can propagate. Due to its high harmonic number ion heating is not expected, but electrons can be heated by the evanescent field, which decays rapidly with the distance from the antenna, and/or by the minor propagating

author'se-mail: rwata@fusion.k.u-tokyo.ac.jp component. Direct current drive by the RF wave is not expected, because the wave excitation has no preferable toroidal direction. Thus, we expect only pressure related current drive mechanisms, such as bootstrap current and asymmetric phase space for confined orbits. The present RF injection experiments provide a unique opportunity for studying the current drive mechanism. In this paper, noninductive start-up experiments using the RF and the EC powers is reported. We have succeeded in sustaining the ECH start-up plasma by the RF power alone, after turning off the ECH power.

Figure 1 shows two typical ECH $(3 \mathrm{~kW})$ start-up discharge types in deuterium plasma. In one discharge (blue), the ECH produced the plasma current, and a current jump occurred $(t \sim 40 \mathrm{~ms})$. In the other discharge (red), a current jump occured just after injection of the RF power $(11 \mathrm{~kW})$ at $t=31 \mathrm{~ms}$. After the ECH power was turned off ( $t=41 \mathrm{~ms})$, the current was sustained by RF heating alone for $40 \mathrm{~ms}$. During the current jump, the high emission region of the total plasma light monitored with the CCD camera shrank toward the midplane. During the sustainment phase, the plasma current increased gradually, and the plasma image barely changed.

While the current jump influences the line integrated electron density at $R=0.28 \mathrm{~m}$ (NL) in both discharges (Fig. 1 (b)), $\mathrm{H}_{\alpha} / \mathrm{D}_{\alpha}$ increased just after the RF power injection, suggesting high recycling at the RF antenna (Fig. 1 (c)). Judging from Fig. 2, the integration lengths are $\sim 0.7 \mathrm{~m}$. Sustaining the plasma current by ECH power 


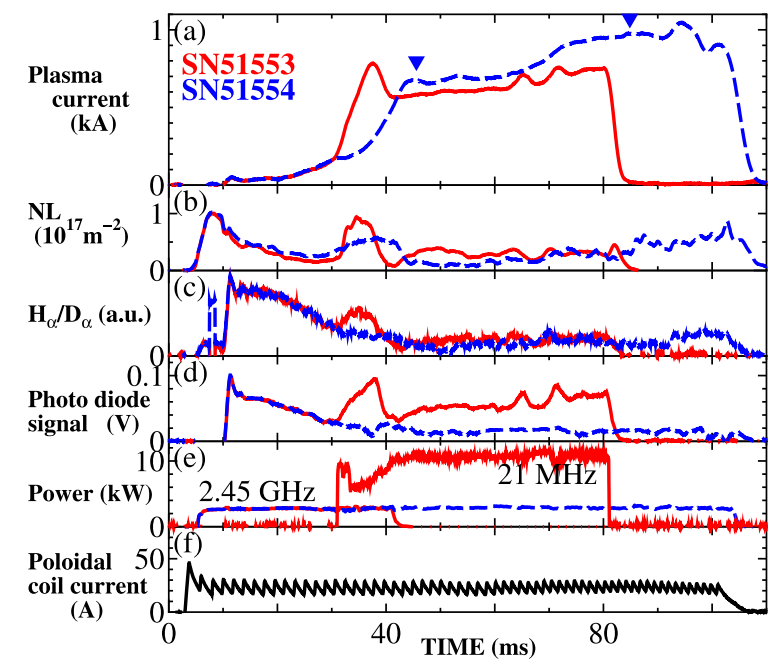

Fig. 1 Discharge waveforms of RF-sustained (red solid line, SN51553) and ECH-sustained (blue dashed line, SN51554) plasmas; (a) the plasma current, (b) the line integrated electron density NL at $R=0.28 \mathrm{~m}$ line (major radius), (c) the $\mathrm{H}_{\alpha} / \mathrm{D}_{\alpha}$ emission, (d) total light measured by photo diode, (e) ECH and RF net powers measured in the transmission line, and (f) poloidal coil current. The typical radiation RF power was $30 \%$ of the net power. Triangles in (a) indicate the timings of additional gas puffs to the ECH-sustained plasma.

alone requires additional gas puffs (triangles in Fig. 1 (a)); without gas puffs the current drops at some time that depends on the initial filling pressure. On the other hand, the RF-sustained plasma does not require additional gas puffs. At the sustainment phase, the line averaged density was $6 \times 10^{16} \mathrm{~m}^{-3}$. These results suggest low recycling for ECH-plasma and high recycling for RF-plasma. The intensity of total light measured by a photo diode (AXUV, IRD Inc.) increased during the RF injection (Fig. 1(d)). The vertical field was kept constant during discharge. Note that the sawtooth waveform shown in Fig. 1 (f) was filtered out inside the vacuum vessel due to its eddy current.

Figures 2 (a) and (b) show a comparison of the profiles of the RF- and ECH-sustained plasmas. The profiles were obtained by Abel inversion of CCD camera images and are normalized by the maximum emissivity for each case. The CCD camera images reflect visible emission, including line emissions from hydrogen/deuterium and light impurities. According to visible spectrum measurements, the relative intensities of those lines between RF- and the EC-sustained plasmas are not singnificantly different. In addition, the intensities of $\mathrm{H}_{\alpha} / \mathrm{D}_{\alpha}$ and total radiation power increase with the injected power. Therefore, the increased emissivity in the RF-sustained plasma can be attributed to its higher recycling and to the enhanced impurity influx due to the larger injected power. According to the profiles, the major radii of both plasmas are about $0.45 \mathrm{~m}$. The difference in the emissivity profiles between RF- and ECH-
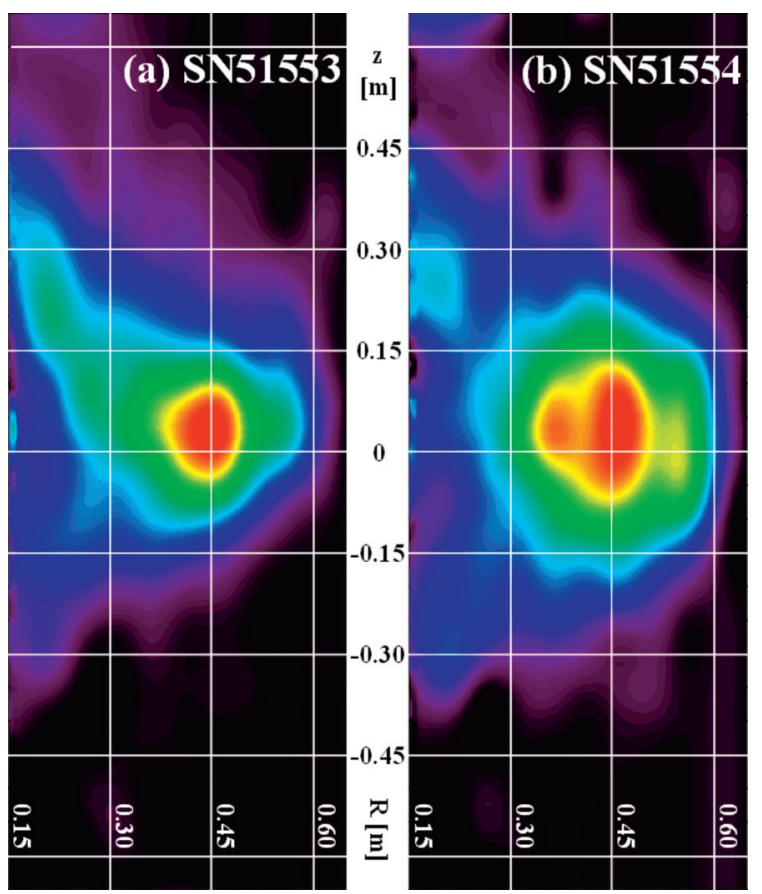

Fig. 2 Normalized emissivities of total plasma light at $44.5 \mathrm{~ms}$ for the same discharges as shown in Fig. 1. (a) RF- and (b) ECH-sustained plasmas. Red indicates bright region, and black indicates dark region.

sustained plasmas is maintained until the end of the RF pulse. Compared with the ECH-sustained plasma, the RFsustained plasma is brighter by a factor of three and its profile is more peaked.

To induce a current jump and sustain the plasma current, the RF power must exceed a certain level (i.e., power threshold). Figure 3 (a) shows the sustainment time (after the turn off of the ECH power) as a function of the net RF power. Below the threshold we cannot sustain the plasma current by RF power alone. Above that threshold, in most discharges, the current was sustained until the end of the $\mathrm{RF}$ pulse ( $8 \mathrm{~ms})$. It is remarkable that the power threshold for hydrogen $(\sim 17 \mathrm{~kW})$ is higher than that for deuterium $(\sim 10 \mathrm{~kW})$. The sustained current does not depend on the RF power, and is similar to the current at the end of the ECH pulse, indicated by the filled symbols in Fig. 3 (b). The same power independence was observed for ECH sustained plasmas [3]. On the other hand, the peak currents at the current jump depends on the injected RF power as indicated by open symbols in Fig. 3 (b).

The operational window for the current sustainment by $\mathrm{RF}$ power alone is narrower than that by $\mathrm{ECH}$ power alone. When the power is well above the threshold the current jump is interrupted, and the plasma current drops before the end of the ECH pulse (see data points with 0 sustainment time in Fig. 3 (a)). In these cases, the magnetic fluctuations $(1-10 \mathrm{kHz})$ increased just before $(0-2 \mathrm{~ms})$ the current drops. We have not succeeded in sustaining the 


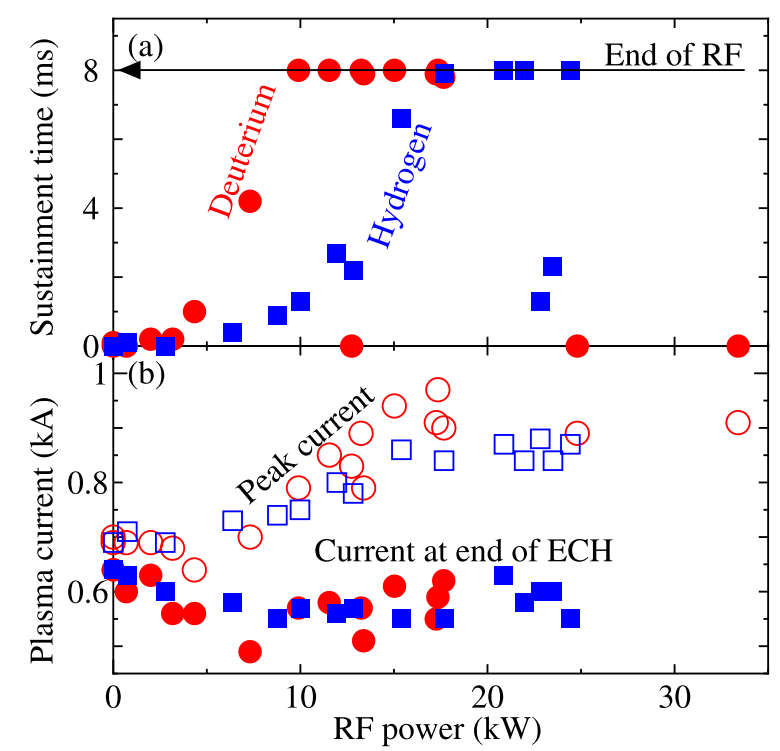

Fig. 3 Comparison of hydrogen (blue squares) and deuterium (red circles) plasmas. (a) shows the dependence of the sustainment time on the RF heating power, and (b) shows plasma currents at maximum (open symbols) and at the end of ECH injection (filled symbols). The ECH startup power was fixed at $4 \mathrm{~kW}$ for these discharges. In these comparison experiments, the RF injection period was fixed at $20 \mathrm{~ms}$. current when either RF or ECH power was too weak. In addition, the sustainment was achieved when the RF was injected into the plasma before a current jump. When we injected the RF power after a current jump was induced by the ECH, the plasma current drops after the ECH was turned off. The current jump is a dynamical process, where magnetic reconnections are expected to form closed magnetic surfaces [4]. In addition, the normalized pressure $\beta_{\mathrm{p}}$ can be high in these plasmas, due to low plasma current. These situations would trigger various MHD instabilities. One candidate method for controlling the dynamics and $\beta_{\mathrm{p}}$ is to adjust the vertical field waveform. Further experiments and analysis are necessary for studying the instabilities and for establishing a reliable start-up scenario.

In summary, RF (in ICRF) current sustainment was demonstrated for the first time in ST start-up experiments. RF can be a candidate tool to start-up the ST reactor.

This work was supported by the Japan Society for the Promotion of Science (JSPS) under Grant-in-Aid for Scientific Research No. 16106013, and NIFS Collaborative Research Program No. NIFS07KUTR021.

[1] C.B. Forest et al., Phys. Rev. Lett. 68, 3559 (1992).

[2] T. Maekawa et al., Nucl. Fusion 45, 1439 (2005).

[3] J. Sugiyama et al., Plasma Fusion Res. 3, 026 (2008).

[4] T. Yoshinaga et al., Phys. Rev. Lett. 96, 125005 (2006). 